\title{
Étude statistique sur les caractéristiques à débit partiel des pompes centrifuges et sur la détermination approximative du débit critique
}

\author{
Statistical Study of partial flow characteristics \\ of centrifugal pumps and of approximate determination \\ of the critical flow \\ R. Rey \\ E.N.S.A.M. Paris \\ P. Guiton \\ e.r. Bergeron S.A. Paris \\ Y. Kermarec \\ C.E.T.I.M. Nantes \\ G. Vullioud \\ Atelier des Charmilles, Genève
}

\section{Introduction}

Toutes les turbomachines fournissant de l'énergie au fluide qui les traversent (pompes, pompes-turbines, ventilateurs, compresseurs) présentent un débit critique $Q_{K}^{(1)}$ quand on réduit leur débit $Q$ (à vitesse angulaire $\omega$ constante) en partant du débit de rendement maximal $Q_{M}$.

Pour $Q<Q_{K}$, l'écoulement débitant ne remplit plus simultanément la totalité des canaux de la roue et il se produit des "courants de recirculation" aux frontières de celle-ci.

La figure 1 les schématise pour une roue de pompe centrifuge.

Le passage des zones de fonctionnement normal aux zones de fonctionnement avec recirculations

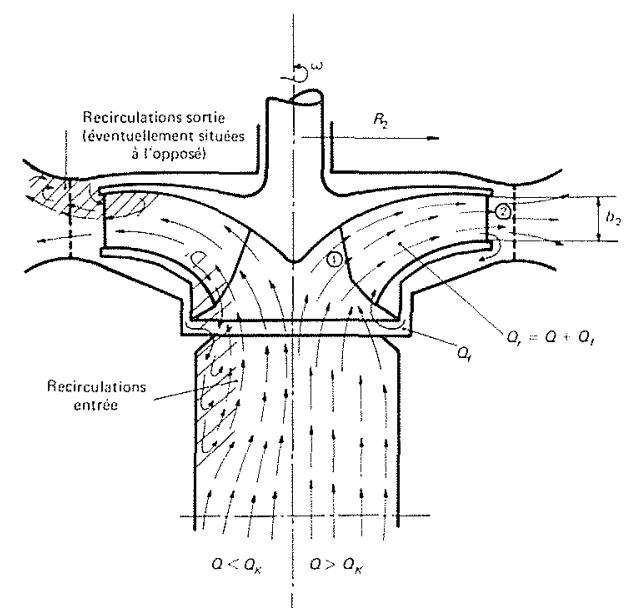

Figure 1-Comparaison entre un écoulement bien organisé (partie droite) et un écoulement avec recirculations (partie gauche).

(1) voir notations à la fin de cet exposé s'effectue, suivant la majorité des observateurs, de manière assez brusque quand on franchit ce débit critique.

En fait, ces recirculations se produisent à l'entrée de la roue pour $Q<Q_{K 1}$ et à la sortie de la roue pour $Q<Q_{K 2}$ mais $Q_{K 1}$ et $Q_{K 2}$ sont en général suffisamment proches l'un de l'autre pour être confondus en une seule valeur $Q_{K}$. Ces recirculations entrainent diverses perturbations, plus ou moins gênantes, dans le fonctionnement de la machine si bien que, pour beaucoup d'entre elles, on s'interdit de fonctionner à un débit inférieur au débit critique. C'est le cas des compresseurs et aussi en général des ventilateurs et des pompes de grande rapidité spécifique $\Omega_{M}$ (pompes hélices ou hélico-centrifuges rapides).

Par contre pour les pompes centrifuges (qui correspondent à $\Omega_{M} \leqslant 2$ environ) on exige de plus en plus le fonctionnement à des débits très inférieurs au débit critique.

Dans toutes les grosses unités de production (raffineries, centrales thermiques classiques ou nucléaires, etc.) ces pompes centrifuges "assurent l'intendance" et doivent être des auxiliaires souples et fiables qui doivent suivre sans défaillance les variations de production.

Par exemple, s'il y a 2 pompes fonctionnant en parallèle et que le débit total nécessaire tombe en dessous de $0,5 Q_{M}$, on continuera par prudence à les faire fonctionner toutes deux à débit réduit plutôt que d'en arrêter une; ceci pour se parer contre un arrêt accidentel de celle restant en service.

Il en résulte des exigences particulières sur la forme des courbes caractéristiques $H(Q)$ et $P(Q)$ depuis $Q=0$ jusqu'à $Q=Q_{M}$.

Par exemple les formes de caractéristiques $a, b$ et $c$ de la figure 2 ne seront pas acceptées, la forme $d$, continuellement descendante, seule l'étant dans bien des cas. 

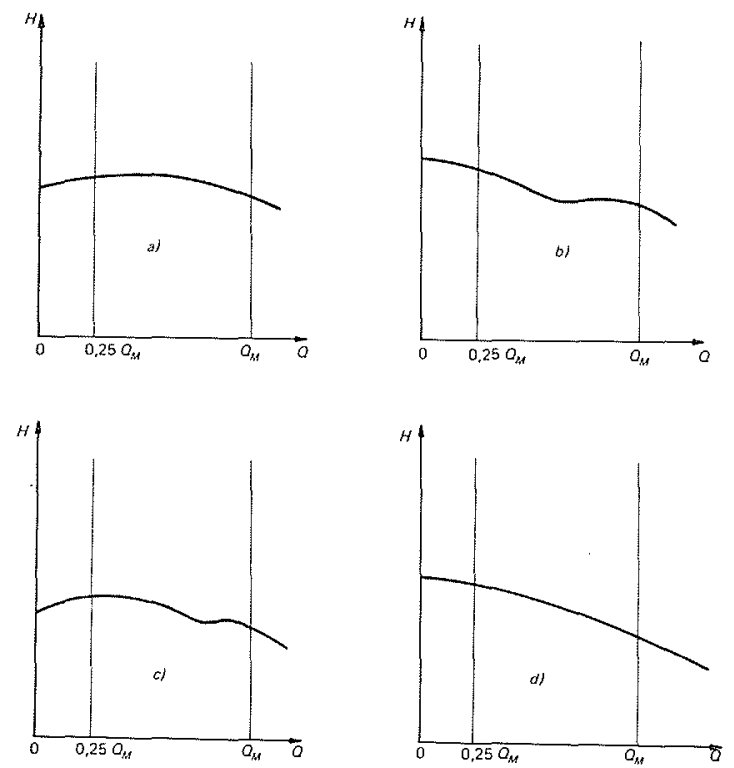

Figure 2-Différentes allures de courbes caractéristiques $H=f(Q)$.

Ces formes de caractéristiques dépendent de beaucoup de paramètres géométriques entre $0,25 Q_{M}$ et $Q_{M}$.

Elles dépendent en particulier de la position de $Q_{K}$, position que l'on peut constater, mais que l'on ne maîtrise pas encore.

Elles dépendent, en outre, de paramètres géométriques encore beaucoup plus nombreux entre $Q=0$ et $0,25 Q_{M}$ environ comme l'expérience le montre.

En réalité chaque pompe est le résultat d'un compromis et suivant qu'on mette en avant le rendement, le prix, l'encombrement, la forme des courbes caractéristiques, la possibilité de marche en sur-débit, l'absence de fluctuations de pression, etc., on aboutit à des résultats différents comme le montrent de nombreux exemples.

Les limitations imposées sur la hauteur à débit nul et/ou sur la puissance à débit nul, peuvent, entre autres, être des éléments déterminants.

Il est vrai que chaque constructeur ayant ses traditions de tracé, ses habitudes conscientes ou non, son expérience et même son style propre, sait souvent à l'avance dans quelle "fourchette" il va tomber pour un choix donné.

Mais comment généraliser?

Pour mieux se rendre compte de l'état des choses, nous avons réuni, au sein du sous-groupe $1^{(2)}$, des courbes caractéristiques (satisfaisantes ou non) des 6 constructeurs $^{(3)}$ qui y sont représentés.

(2) Sous-groupe 1c de la SHF : Etude de l'influence des divers paramètres géométriques et hydrauliques sur les caractéristiques $\bar{H}(\bar{Q})$ et $\bar{P}(\bar{Q})$ à débit partiel des pompes et ventilateurs.

(3) - Ateliers des Charmilles (Genève)

- Alsthom-Atlantique (Rateau-La Courneuve)

- Bergeron S.A. (Paris)

- C.C.M. Sulzer (Mantes)

- Jeumont-Schneider (Pûteaux)

- Pompes Guinard (Annecy)
Ceci nous a permis d'aboutir à une véritable "population" de caractéristiques. Nous avons cherché a en faire une statistique à partir de points représentatifs de chaque caractéristique.

Comme nous allons le voir, nous pourrons constater une grande dispersion de ces points, mais avec cependant, pour les caractéristiques "satisfaisantes" des plages de regroupement caractérisées permettant de délimiter en gros des "fourchettes" globales.

\section{Classifications des pompes - Traitement des données}

Comme nous venons de le dire, les pompes étudiées représentent en échantillon des fabrications de l'ensemble des constructeurs représentés au sein du sous-groupe $1 c$ de la SHF.

Les résultats d'essais accompagnés des valeurs de $\omega$ et $D_{2}$ (et moins systématiquement de $b_{2}$ ), furent regroupés et rendus anonymes par les soins de l'un d'entre nous, Monsieur Kermarec, du CETIM de Nantes.

Ces pompes se présentent en plusieurs groupes caractérisés par le type de construction : roue à 1 ouie, à 2 ouies, volute ou diffuseur, à bulbe, etc. (Fig. 3).

Pour que les statistiques ne soient pas trop faussées par des pompes ayant des pertes trop élevées, il a été convenu de ne prendre en compte que des pompes ayant un rendement global $\eta$ supérieur à 0,8 pour des rapidités spécifiques $\Omega_{M}$ comprises entre 0,2 et 2 ; en dehors de cette plage, on pouvait abaisser cette limite de $\eta$ jusqu'à 0,7 .

Les machines ainsi retenues, sont classées en 5 sous groupes principaux en fonction de la forme de la caractéristique $H(Q)$ (voir Fig. 3), caractéristique descendante, descendante avec bosse, avec creux et contrepente, avec bosse et creux, (ces creux ou bosses pour $Q>0,25 Q_{M}$ ) et enfin creux et/ou bosse pour $Q<0,25 Q_{M}$, ce dernier défaut étant souvent moins génant que les autres.

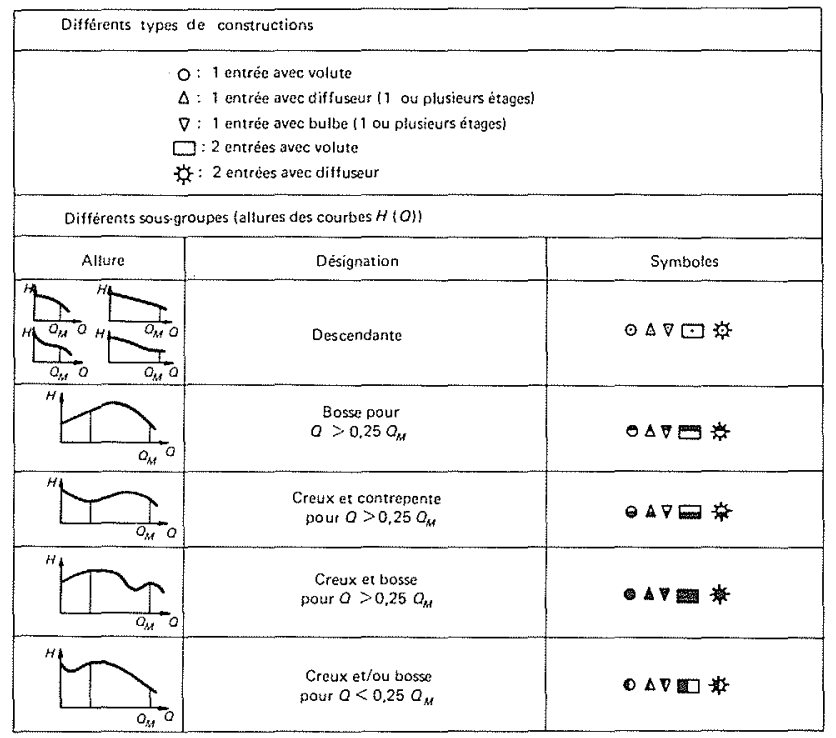

Figure 3 - Classification en fonction du type de construction et de la forme de la caractéristique $H=f(Q)$. 


\section{EVOLUTION DES CARACTERISTIQUES DANS LES ZONES A DEBIT PARTIEL}

La rapidité spécifique $\Omega_{M}$ ou le coefficient de hauteur totale $\psi_{M}$ au point de rendement maximal $M$ sont les paramètres généralement retenus pour classifier les pompes. Nous les avons également choisis pour classifier les performances à débit partiel.

Plus particulièrement, c'est le coefficient $\psi_{M}$ qui a été retenu comme paramètre de référence pour avoir donné des résultats moins dispersés que $\Omega_{M}$.

Du reste le graphique $\psi_{M}\left(\Omega_{M}\right)$ des pompes centrifuges que nous avons étudiées (Fig. $4 a$ ) permet de passer de l'un des paramètres à l'autre. Ce graphique en recoupe bien d'autres du même genre ou qui en dérivent, publiés par différents auteurs (Fig. $4 b$ ).

$\mathrm{La}$ figure 5 définit les caractéristiques ayant été étudiées dans notre statistique :

$$
\frac{H_{25}}{H_{M}}, \frac{H_{0}}{H_{M}}, \frac{H_{T_{0}}}{H_{M}}, \frac{P_{25}}{P_{M}}, \frac{P_{0}}{P_{M}}
$$

Cette représentation des courbes en valeurs relatives:

$$
\frac{H}{H_{M}}\left(\frac{Q}{Q_{M}}\right) \frac{P}{P_{M}}\left(\frac{Q}{Q_{M}}\right) \frac{\eta}{\eta_{M}}\left(\frac{Q}{Q_{M}}\right)
$$

s'est montrée particulièrement souple et appropriée à nos travaux. Le point $M$ a étê soigneusement déterminé par la méthode de la médiane ${ }^{(4)}$.

Rappelons que la pente relative de la courbe $H(Q)$ en $M$, donnée par l'ordonnée $H_{T_{0}} / H_{M}$ de la figure 5 a une grande importance, non seulement sur l'allure des courbes $H / H_{M}\left(Q / Q_{M}\right)$ et $P / P_{M}\left(Q / Q_{M}\right)$, mais aussi sur les conditions et possibilités d'utilisation de la pompe. D'autre part, la valeur qui en découle pour l'ordonnée $P_{T_{0}} / P_{M}$ (voir nota ${ }^{(4)}$ ) divise les pompes centrifuges en "centrifuges lentes" pour $P_{T_{0}} / P_{M}<1$ (puissance croissante avec le débit en $M$ ) et en "centrifuges rapides" pour $P_{T_{0}} / P_{M}>1$ (puissance déerolssante avec le débit en $M$ ).

Ce rappel est utile à notre étude statistique : Les résultats de celle-ci, qui portent sur plus de 60 pompes centrifuges diffèrentes, sont donnés sous la forme des 5 graphiques suivants :

- Figure 6a: $\frac{H_{25}}{H_{M}}\left(\psi_{M}\right)$, Figure $6 b: \frac{H_{0}}{H_{M}}\left(\psi_{M}\right)$,

Figure $6 c: \frac{H_{T_{0}}}{H_{M}}\left(\psi_{M}\right)$

- Figure 7a $: \frac{P_{25}}{P_{M}}\left(\psi_{M}\right), \quad$ Figure $7 b: \frac{P_{0}}{P_{M}}\left(\psi_{M}\right)$

(4) Nota : Dans ces conditions, on vérifie que si les trois courbes:

$$
H / H_{M}\left(Q / Q_{M}\right), P / P_{M}\left(Q / Q_{M}\right) \text { et } \eta / \eta_{M}\left(Q / Q_{M}\right)
$$

sont bien cohérentes entre elles, la distance entre les points $H_{T_{0}} / H_{M}$ et $P_{T_{0}} / P_{M}$ est égale à 1 dans cette représentation, ce qui se démontre aisément. Ces 3 courbes peuvent ne pas être cohérentes, si elles ont été interpolées indépendamment les unes des autres entre les points d'essai.
Sur ces 5 graphiques, on peut constater que la grande majorité des points correspondant à des pompes à caractéristiques $H(Q)$ "satisfaisantes" se regroupent pour chacun d'eux dans une plage dont on peut déterminer approximativement le contour par des traits mixtes.

La largeur relative de ces plages donne une idée de la dissémination rencontrée sur l'ensemble de la statistique, mais si les frontières supérieures sont assez floues, les frontières inférieures le sont beaucoup moins. En dessous, il n'y a que 1 ou 2 points isolés qu'il faudrait analyser d'avantage.

Il est donc prudent, tant pour l'utilisateur que pour le constructeur, de rester avec une certaine marge au-dessus de ces limites inférieures pour avoir des caractéristiques $H(Q)$ continuellement descendantes.

Ceci ne veut pas dire qu'en dessus, on ait toute garantie, comme le montre le nombre de points entachés de noir que l'on trouve un peu partout sur ces graphiques.

Bien entendu, pour les raisons indiquées au début de cet exposé, les largeurs relatives de plage sont nettement plus grandes pour $Q=0$ que pour $Q=0,25 Q_{M}$, c'est pourquoi nous avons présenté ces dernières en premier. On constate, mais cela est bien connu, que l'augmentation de $P_{0} / P_{M}, P_{25} / P_{M}$ et la diminution de $P_{25}-P_{0} / P_{M}$ vont de pair respectivement avec l'augmentation de $H_{0} / H_{M}, H_{25} / H_{M}$ et $H_{0}-H_{25} / H_{M}$. C'est ce que nous avons mis en évidence sur les graphiques des figures $8 a, 8 b$ et $8 c$.

Il peut en résulter certaines incompatibilités; par exemple, on ne peut pas chercher à réduire à tout prix la puissance à débit nul $P_{0} / P_{M}$ sans risquer de tomber sur une courbe $H(Q)$ non entièrement satisfaisante.

\section{Limite d'apparition des zones perturbées (point $K$ )}

Comme nous l'avons déjà indiqué dans l'introduction, $Q_{K_{1}}$ débit d'apparition des recirculations à l'entrée de la roue ne coïncide pas forcément avec $Q_{K_{2}}$ débit d'apparition des recirculations à la sortie de la roue. En général, ils sont pourtant suffisamment rapprochés pour qu'on puisse les confondre en écrivant $Q_{K_{1}}=Q_{K_{2}}=Q_{K}$.

La localisation de ce point particulier fait l'objet de beaucoup de soins de la part des expérimentateurs, il peut quelquefois être accompagné de l'apparition d'une contre-pente locale ou d'un décrochage plus ou moins accentué des courbes $H(Q)$ et $P(Q)$ (cas 1 , Fig. 9) et plus souvent d'un changement de courbure de cette courbe $P(Q)$ (cas 2, Fig. 9).

En l'absence de recirculations, la courbe $P(Q)$ se prolongerait jusqu'à une valeur très faible pour $Q=0$ comme schématisé en traits mixtes sur la figure 9. Cette valeur ne correspondrait, en effet, qu'à la puissance absorbée par frottements fluide ou autres de l'extérieur du rotor et à celle nécessaire pour faire traverser l'aubage par le seul débit de fuite aux bagues.

Déceller $Q_{K}$ à partir des courbes caractéristiques d'essai est donc aisé, si l'on étudie une pompe manifestant les particularités du cas 1, mais des points de mesure très rapprochés sont alors nécessaires. 


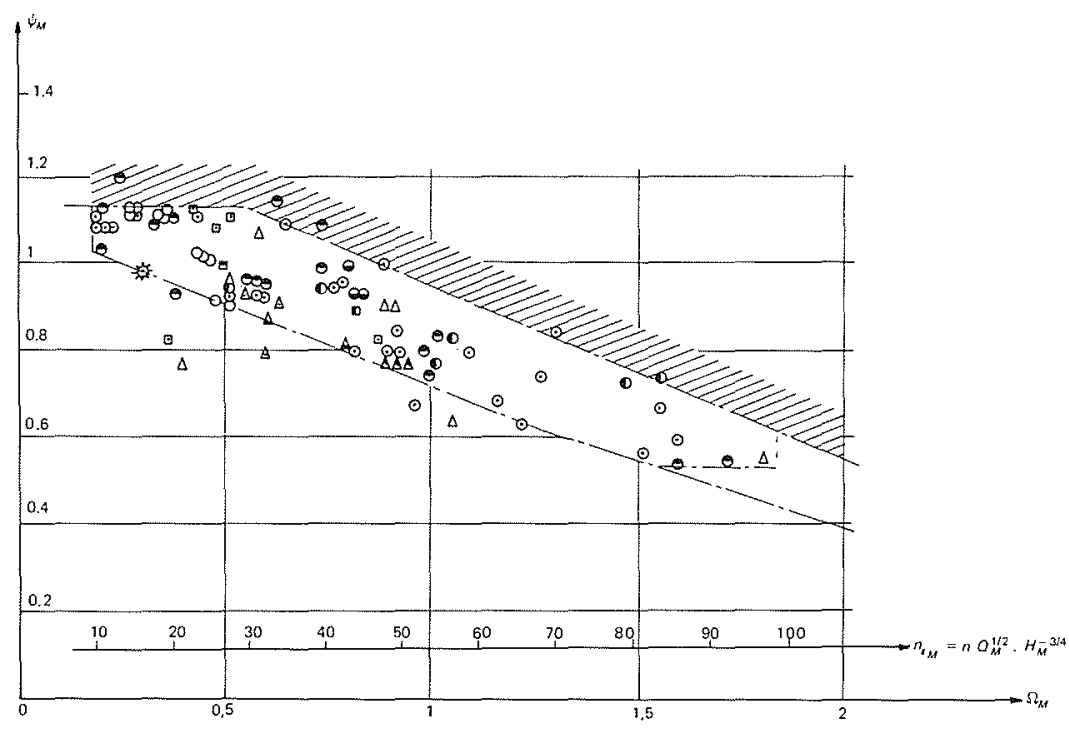

Figure $4 a$ - Evolution du coefficient de hauteur totale en fonction de la rapidité spécifique de la pompe (tous deux calculés au point de rendement maximal).

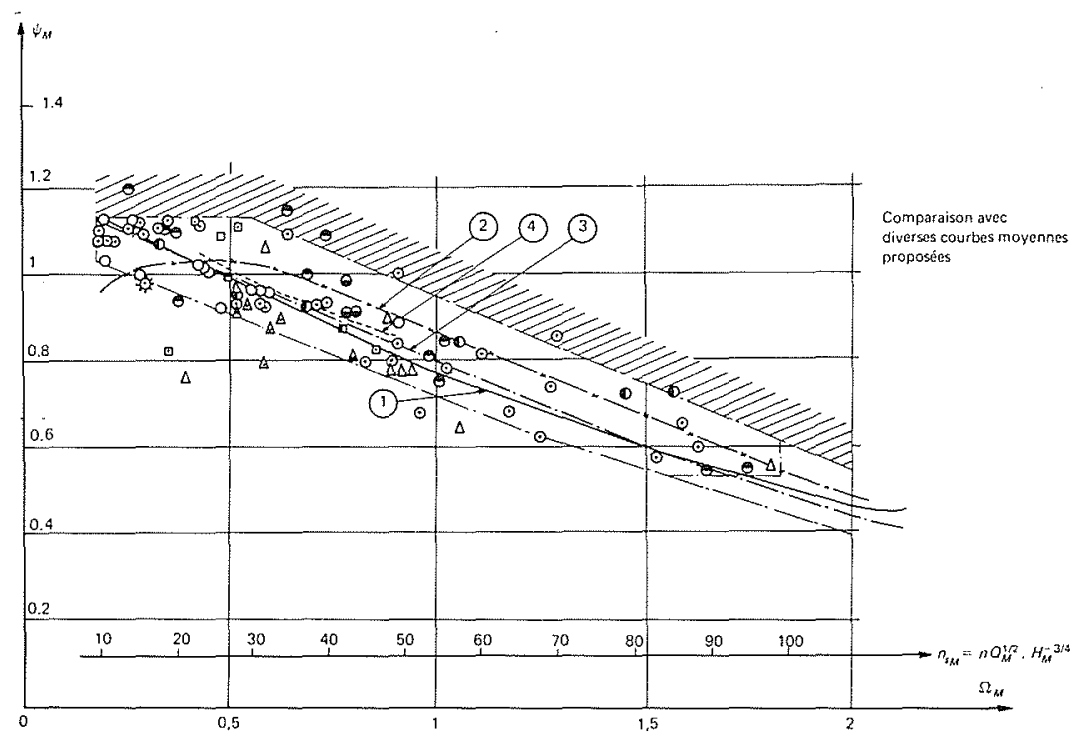

Figure $4 b$ - Evolution du coefficient de hauteur totale en fonction de la rapidité spécifique de la pompe pour diverses courbes moyennes proposées

(1) Déduit de Stepanoff. Pompes centrifuges et pompes hélices.

$$
\text { Figure } V_{Z} K V\left(n_{\mathrm{s}}\right) \text { avec } \psi_{M}=\frac{1}{K V^{2}}
$$

$\longrightarrow$ (2) Déduit du diagramme de Cordier :

$$
\Lambda_{M}\left(\Omega_{M}\right) \quad \text { avec } \quad \Lambda_{M}=\frac{R_{\mathbf{2}}\left(g H_{M}\right)^{1 / 4}}{Q_{M}^{1 / 2}} \text { d'où } \quad \psi_{M}=\frac{2}{\Lambda_{M}^{2} \Omega_{M}^{2}}
$$

(Voir par exemple Csanady Figure 6 avec ' $A=\Lambda$

(3) Déduit de Tsabersky, Acosta, Hanptmann. Fluid flow 2nd Edition,

$$
\text { Figure } 16.26 \text { avec } \psi_{M}=2 \psi \Omega_{M}=N_{s}
$$

(4) Déduit de Graeser : Abaque pour pompes et pompes-turbines réversibles : EPEL. Lausanne 1971. Figure 14 avec $\psi_{M}=\psi_{q i}$ et $\Omega_{M}=2^{3 / 4}, \pi^{1 / 2}, \nu_{R}$. 


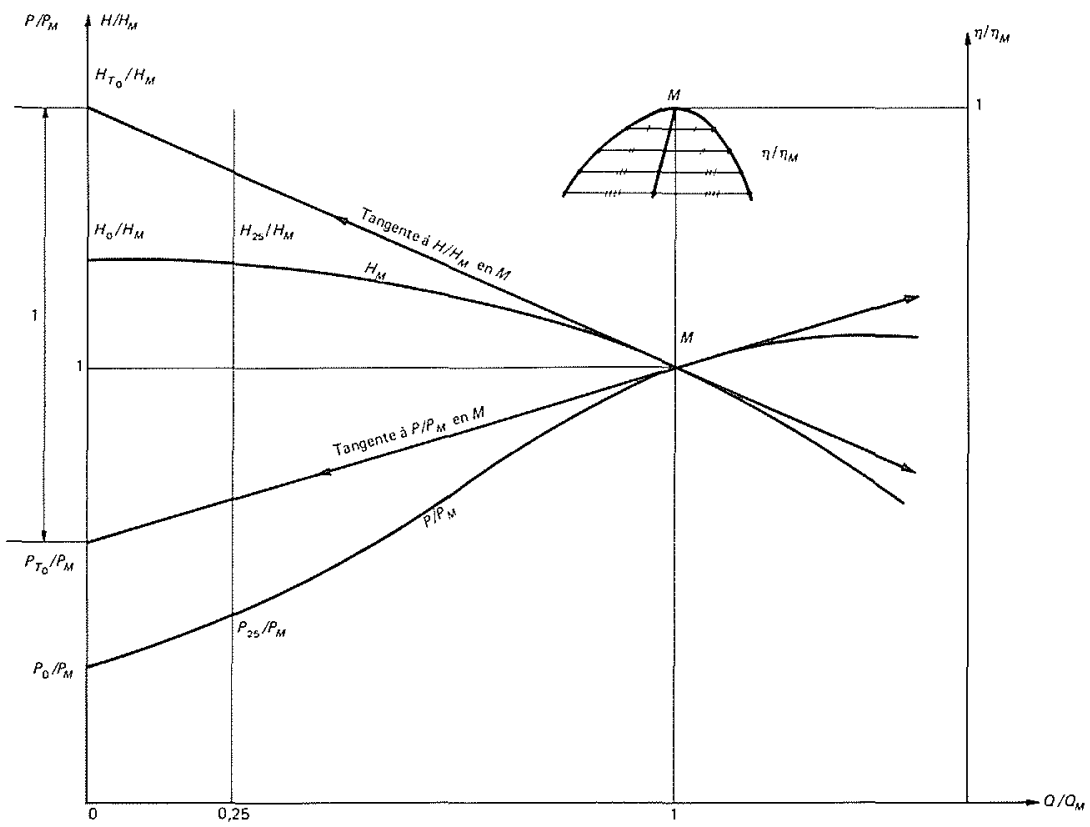

Figure 5 - Définition des divers paramètres étudiés.

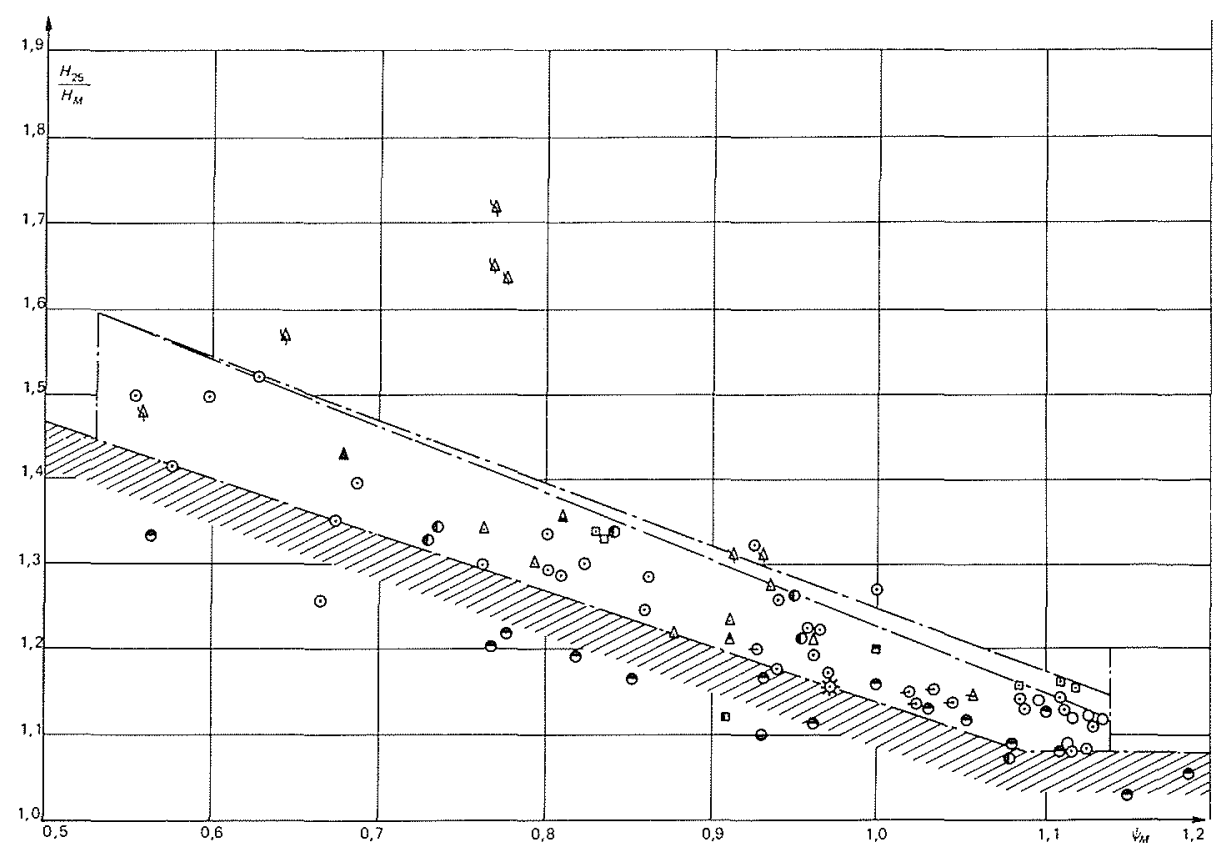

Figure $6 a-$ Evolution de la hauteur à $25 \%$ du débit de rendement maximal en fonction du coefficient de hauteur totale. 


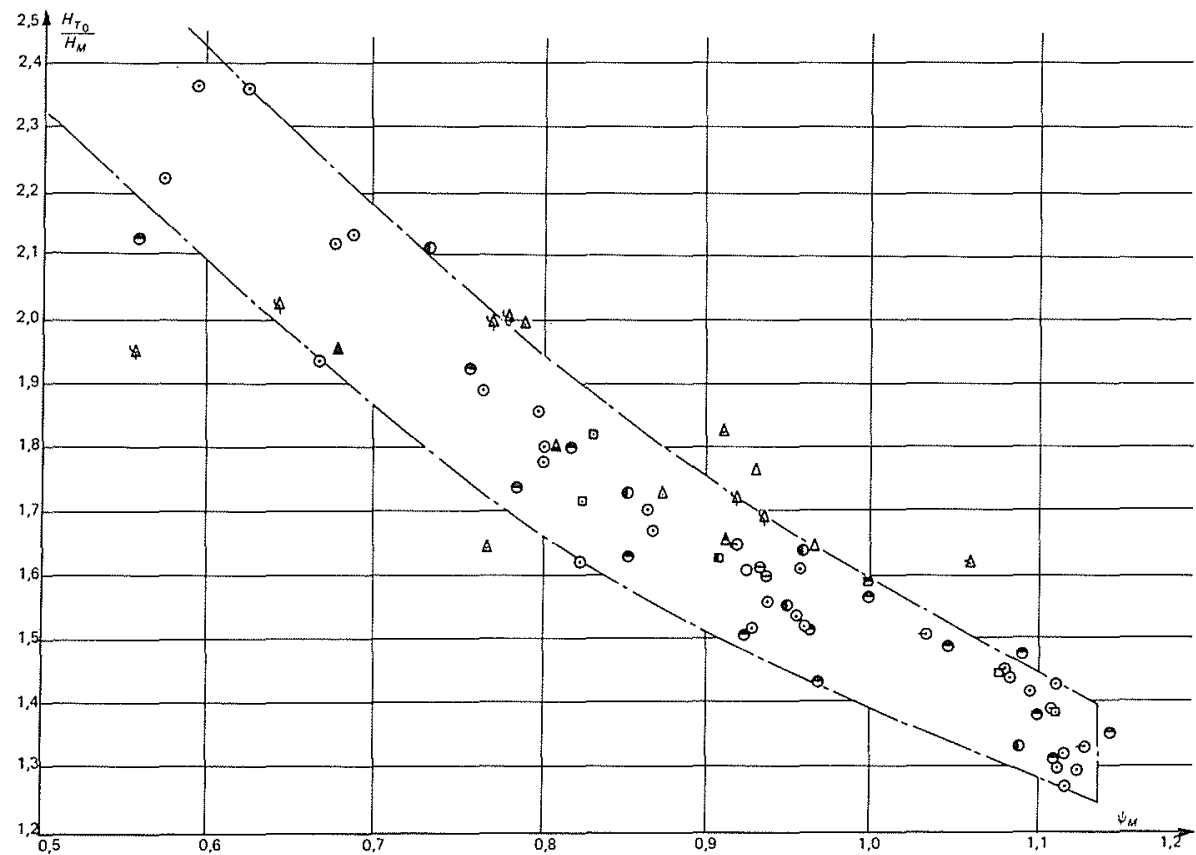

Figure $6 b$ - Evolution de la hauteur à débit nul en fonction du coefficient de hauteur totale.

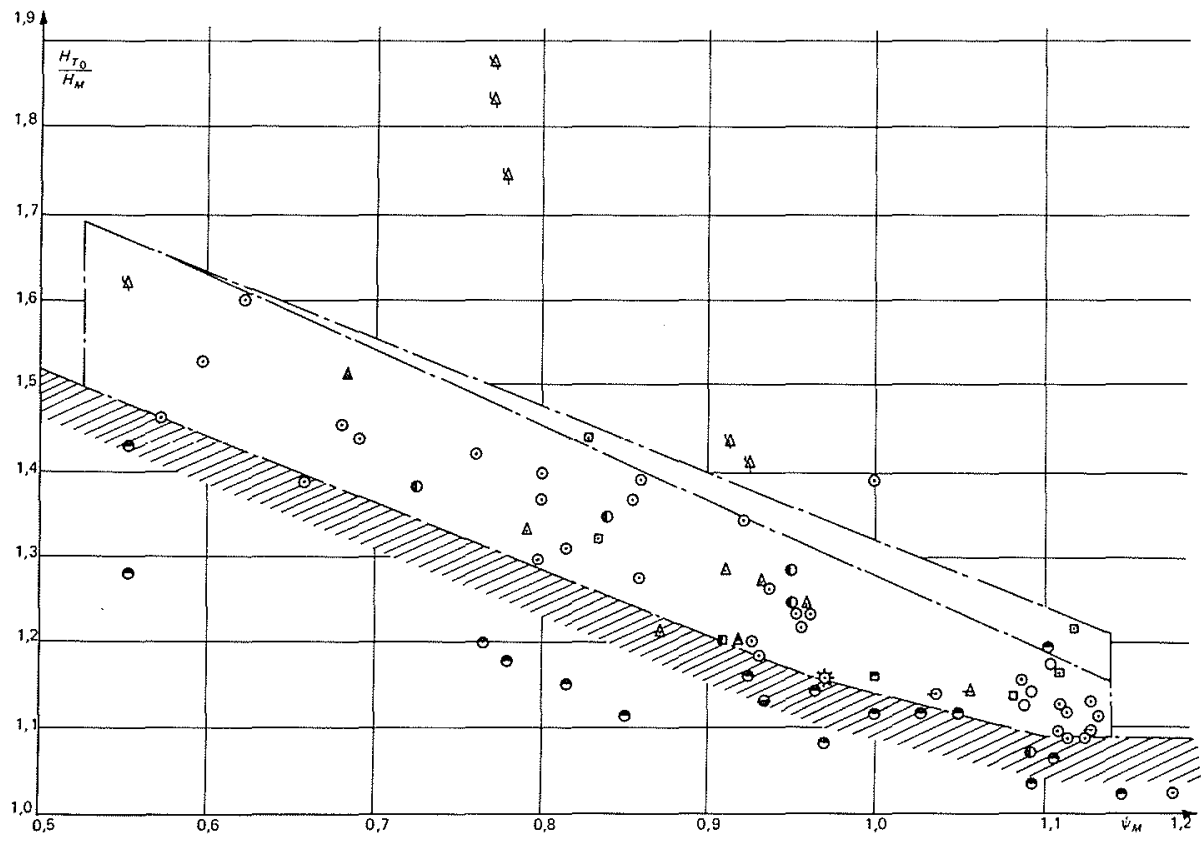

Figure $6 c .-H_{T 0} / H_{M}$ défini à la figure 5. 


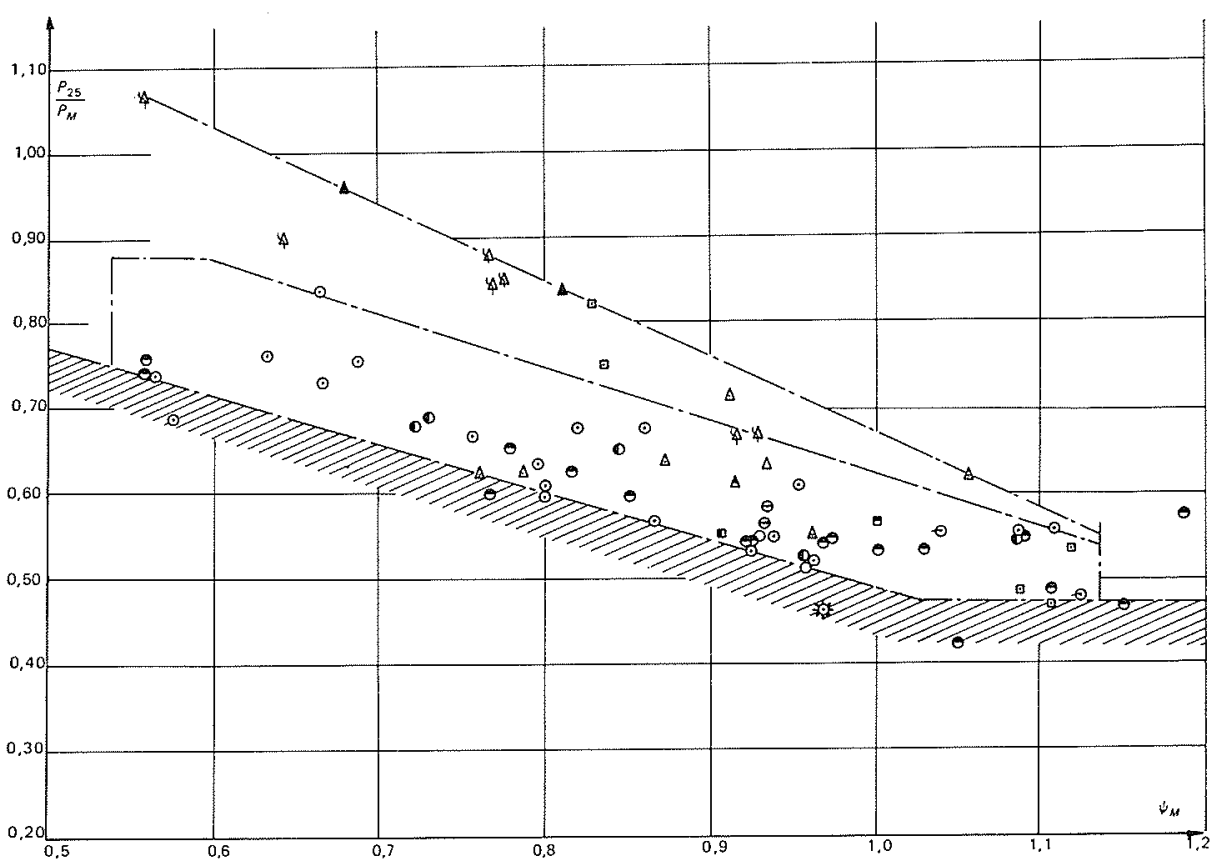

Figure $7 a$ - Evolution de la puissance à $25 \%$ du débit de rendement maximal en fonction du coefficient de hauteur totale.

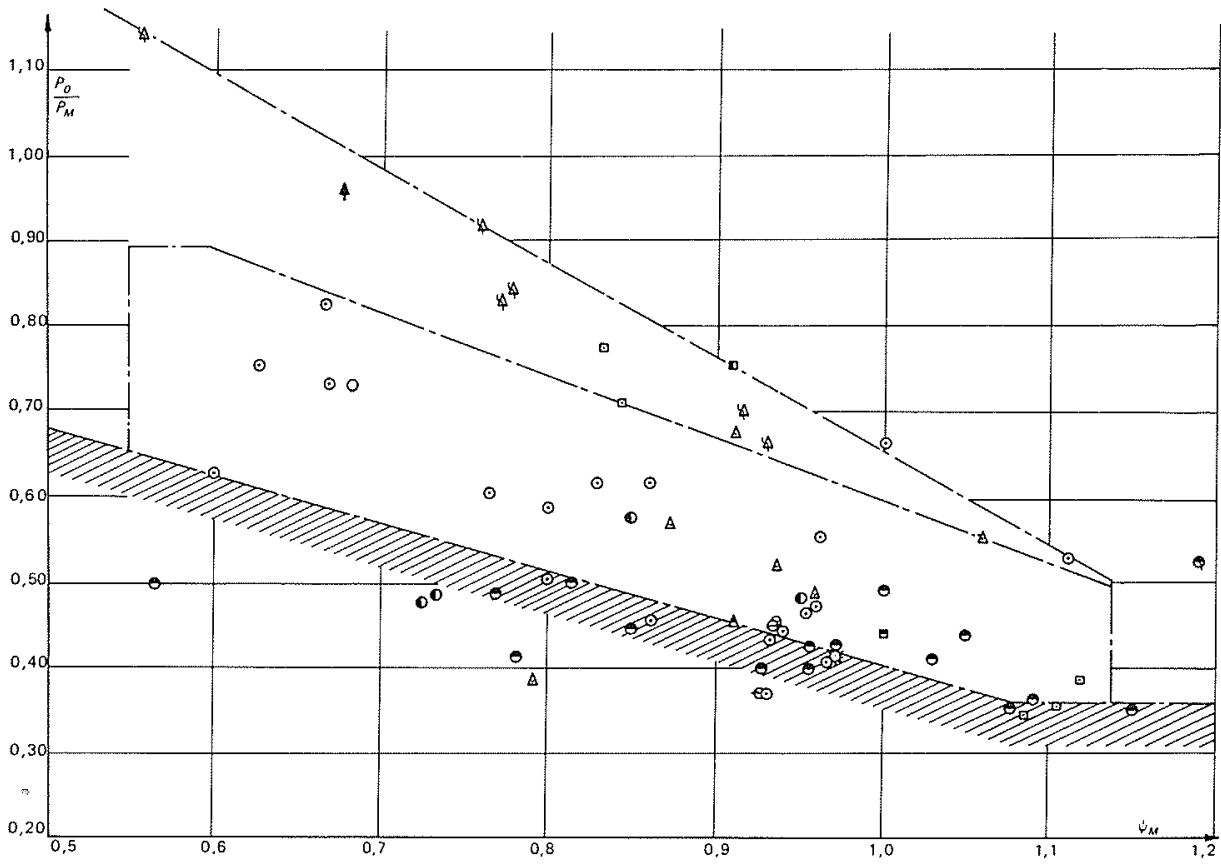

Figure $7 b$ - Evolution de la puissance absorbée à débit nul en fonction du coefficient de hauteur totale. 


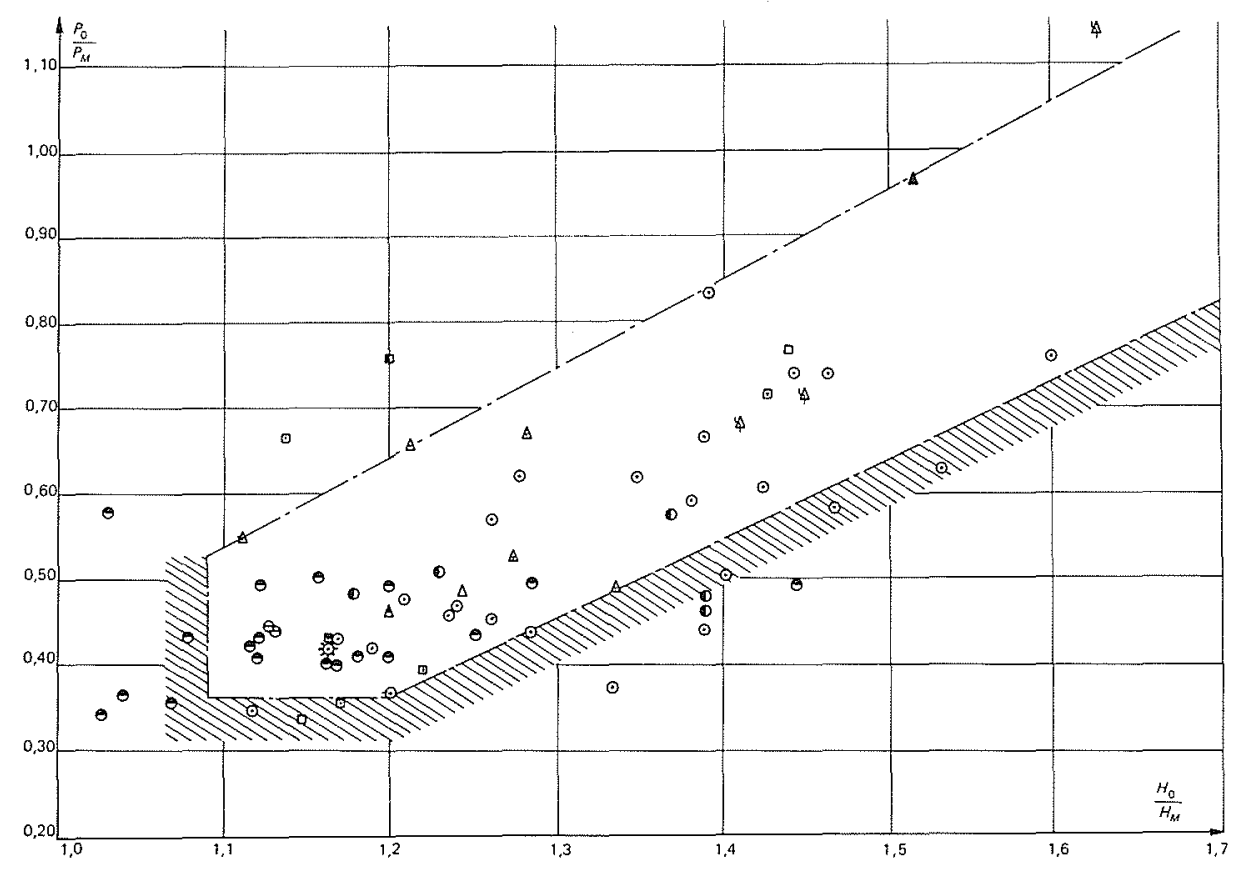

Figure $8 a$ - Evolution de la puissance à débit nul en fonction de la hauteur à débit nul.

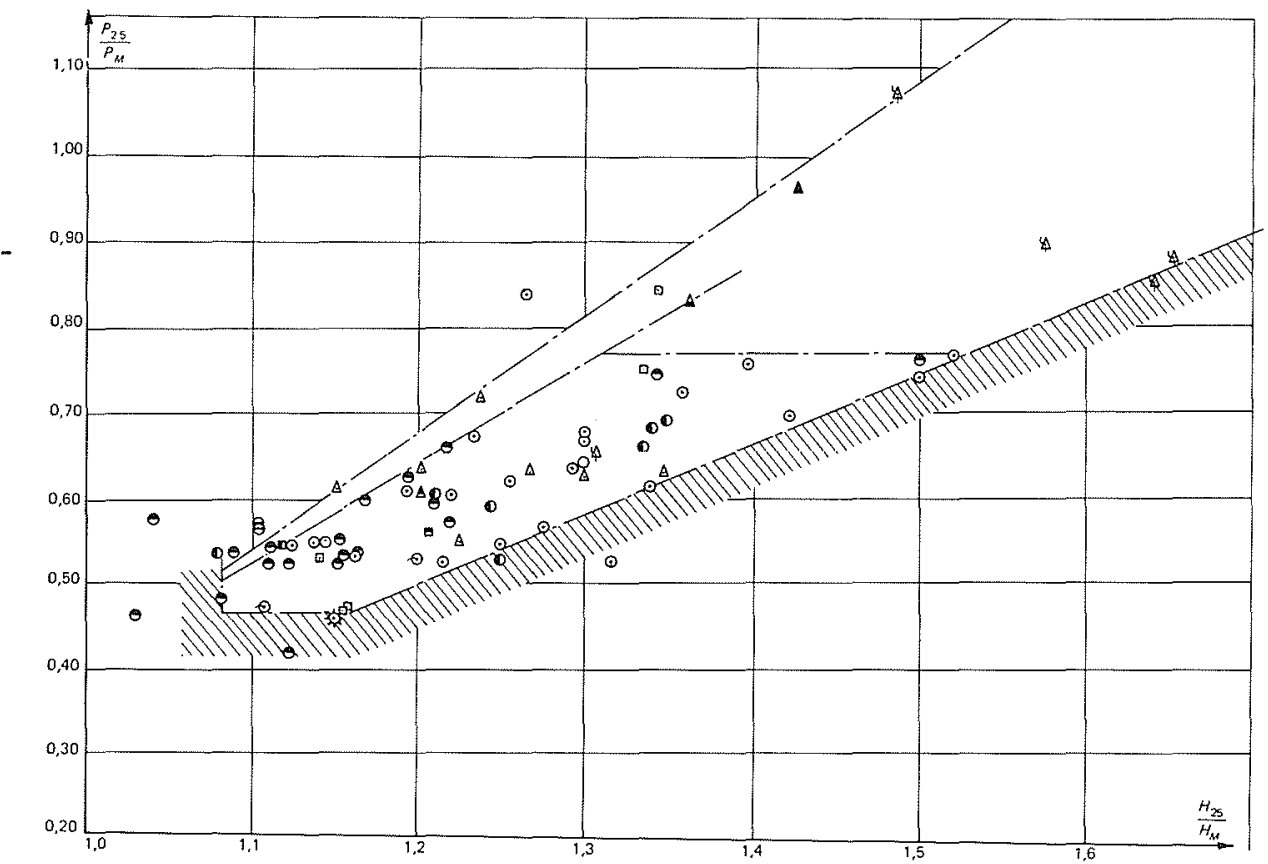

Figure $8 b$ - Evolution de la puissance à $25 \%$ du débit de rendement maximal en fonction de la hauteur à ce même débit. 


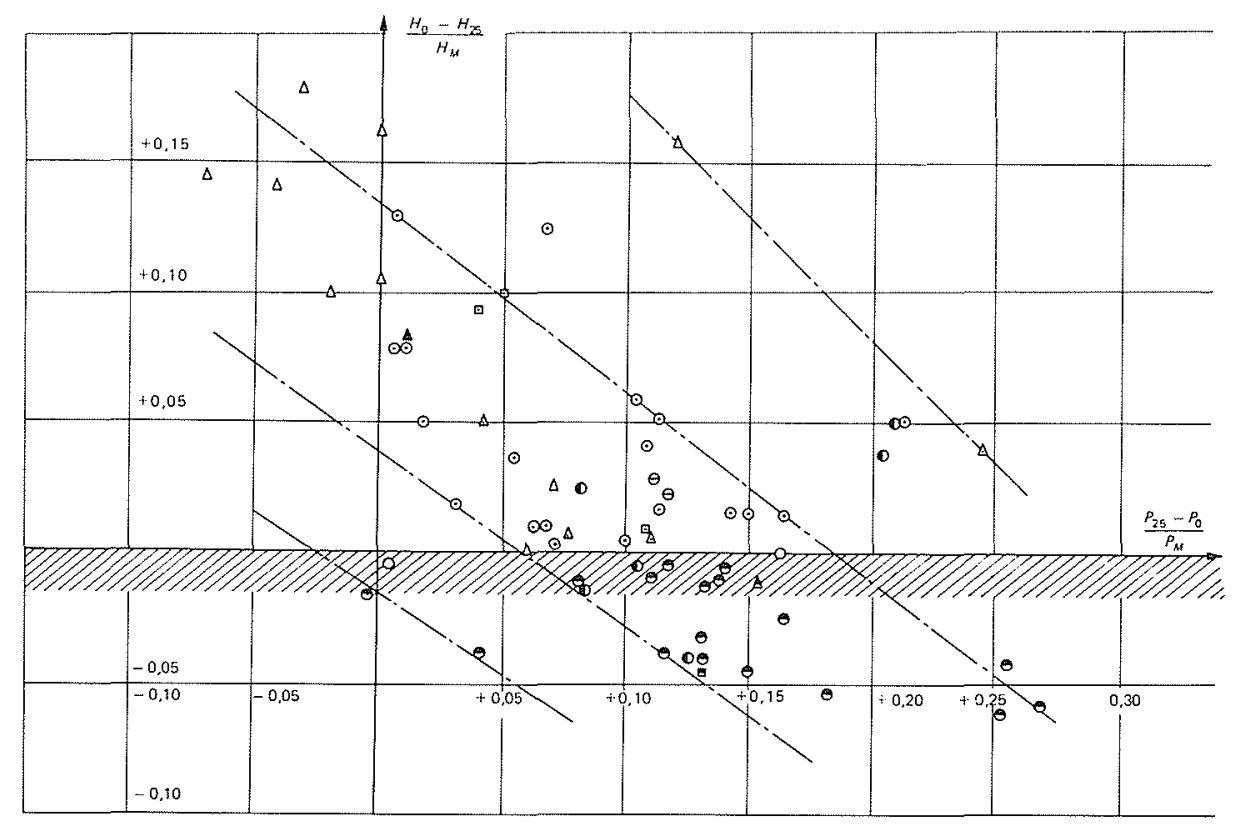

Figure $8 c$ - Fonctionnements en débit partiel.
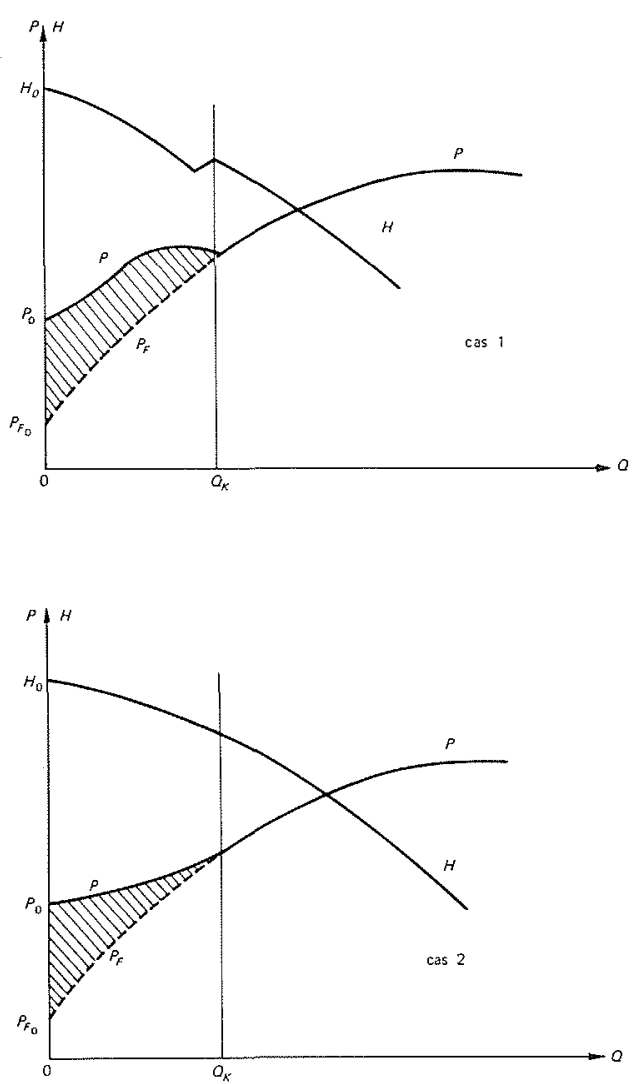

Figure 9 - Définition du débit d'apparition des recirculations $\left(Q_{K}\right)$ et mise en évidence de la puissance liée à celles-ci.
Dans la majorité des cas, la variation de la pente locale de $P(Q)$ n'apparait pas nettement et il faut avoir recours à d'autres méthodes :

\section{a) Techniques de détermination directe de $Q_{K}$}

Nous ne nous y attarderons pas, car ce n'est pas le but de notre exposé. Elles nécessitent des moyens d'essai et des dispositifs spéciaux, ainsi que des aménagements particuliers de la pompe à essayer, ce qui fait qu'elles sont rarement applicables à la majorité des pompes centrifuges industrielles.

En outre, elles nécessitent des espaces libres ou entrefers suffisants et convenablement disposés tant à l'aspiration (détermination de $Q_{K}$ par $Q_{K_{1}}$ ) qu'au refoulement (détermination de $Q_{K}$ par $Q_{K_{2}}$ ). Ce que ne possédent pas la plupart des pompes.

Citons parmi ces techniques : la visualisation par fils colorés, l'évolution en fonction du débit des surpressions à la paroi du tube d'aspiration-dues à la prérotation, l'évolution de la cavitation des aubes en fonction du débit ${ }^{(5)}$. Les discontinuités dans l'évolution des pressions de paroi du stator du diamètre $D_{2}$ en fonction du débit, etc. La variation du bruit est aussi un moyen mais imprécis.

(5) L'un des auteurs, Monsieur Vullioud, a présenté au sousgroupe $1 c$ des photos montrant bien l'évolution de cette cavitation d'aubage au voisinage de $Q_{K}$ pour une pompe centrifuge correspondant au cas 1 de la figure 9 . 


\section{b) Méthodes indirectes de détermination de $Q_{K}$ à partir de la courbe $P(Q)$}

Elles consistent à tracer au mieux, par divers procédés, la courbe $P_{F}$ de la figure 9 cas 2 , et à voir ensuite comment $P-P_{F}$, puissance absorbée due aux recirculations, évolue avec $Q / Q_{M}$ et enfin de définir une valeur conventionnelle de $Q_{K}$ là où $P-P_{F}$ s'annule. Cette valeur sera comparée chaque fois que possible à la valeur $Q_{K_{1}}$ ou $Q_{K_{2}}$ déterminée directement.

Une étude statistique a été entreprise dans ce sens. Pour faciliter le tracé de la courbe $P_{F}$ raccordant la courbe $P$ hors recirculations au point $P_{F_{0}}$ (Fig. 9). il est paru avantageux de passer par la courbe $H t h(Q)$ (ou $H i$ ) (hauteur théorique ou hauteur interne) qui, comme on le sait, est pratiquement une droite pour $Q>Q_{K}$ en l'absence de cavitation.

Pour en arriver à ces résultats, quelques diagrammes statistiques concernant le rendement mécanique $\eta_{m}$ et le rendement volumique $\eta_{v}$ furent utilisés, diagrammes inspirés de Stepanoff que Monsieur Lapray a bien voulu contrôler et complèter sur la base de résultats expérimentaux en sa possession, ces diagrammes sont donnés pour le point de fonctionnement nominal $(M)$. Ils donnent une valeur conventionnelle de ces pertes, suffisante pour cette étude.

Pour chaque pompe, il est aisé de tracer point par point la hauteur théorique ${ }^{(6)} H t h$, qui, si l'on fait abstraction des recirculations, tend expérimentalement vers l'infini pour $Q=0$.

Cette remontée de la courbe $H t h$ met en évidence ces recirculations qui produisent d'autres échanges de moments de qualité de mouvement entre la roue et le stator que ceux du modèle d'Euler (Fig. 10).

Dans la mesure où la courbe Hth $(Q)$ est bien rectiligne dans la zone sans recirculation, l'hypothèse de travail la plus simple ${ }^{(7)}$ est d'admettre qu'en l'absence de recirculations elle se prolongerait par la portion de droite tangente $H_{E}$ jusqu'au point $\mu_{0} U_{2}^{2} / g$ à débit nul. Cette figure met bien en évidence que le débit $Q_{K}$ est celui au dessous duquel le modèle d'Euler ne convient plus. La séparation de ces deux courbes au point $Q_{K}$ est cependant peu nette et ne permet pas de déterminer avec précision $Q_{K}$. Mais on peut maintenant tracer dans une deuxième étape, la puissance liée à ces recirculations $\left(P t h-P_{E}\right)=(\rho Q r) g\left(H t h-H_{E}\right)$ qui décroit pour s'annuler à partir de $Q_{K}$. $\left(P t h-P_{E}\right)=\left(P-P_{F}\right)$ de la figure 9 car $(P-P t h)=\left(P_{F}-P_{E}\right)=P_{F_{0}}=P_{f}+$ $\left(\rho Q_{f}\right)\left(g H_{\Sigma_{0}}\right)$.

$\mathrm{Au}$ cours des analyses faites à l'ENSAM Paris sur un certain nombre de courbes caractéristiques, on a pu constater que ces pertes évoluaient pratiquement presque toujours comme le logarithme du débit, sur une large plage auldelà de $0,25 Q_{M}$.

(6) Rappelons que $(g H t h)$ n'est que la mesure en $J / K g$ de l'énergie communiquée à l'aubage de la roue par unité de masse de liquide la traversant de part en part, ce qui n'a rien de "théorique". C'est pourquoi l'expression hauteur interne est souvent préférée.

(7) On peut envisager d'autres modes de raccordement que la droite tangente $H_{E}$, mais ceci ne change pas le raisonnement.
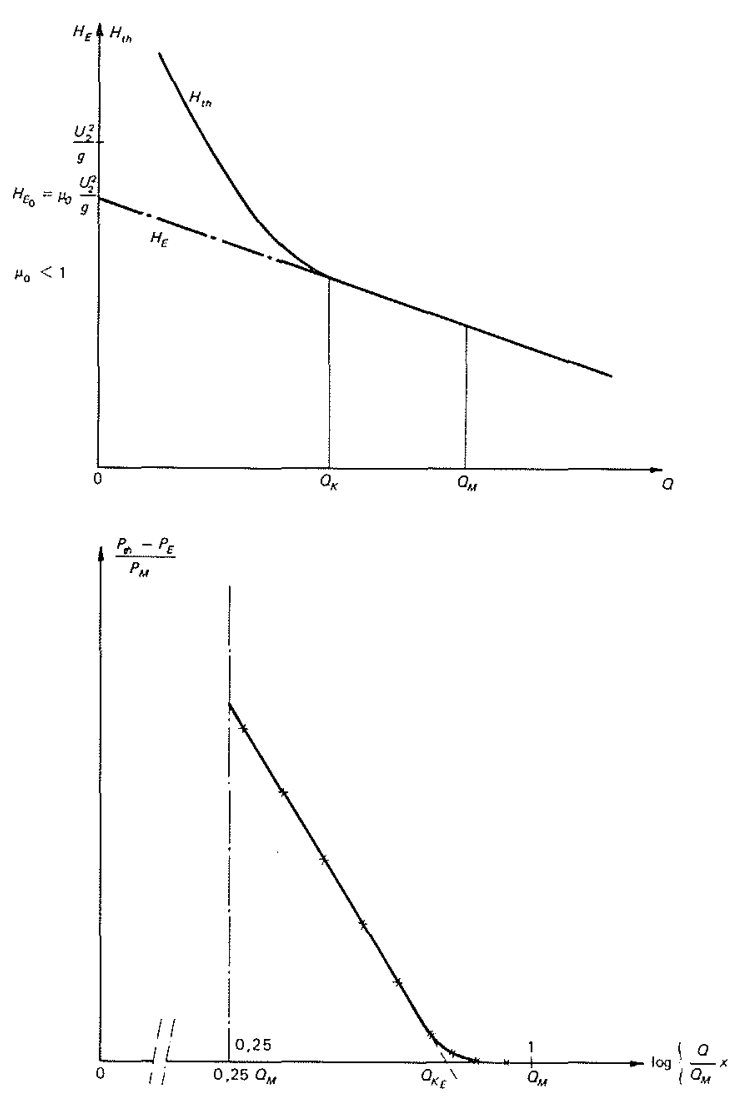

Figure 10 - Divergence entre le modèle d'Euler et la hauteur théorique. Evolution de la puissance dûe aux recirculations avec le débit.

Pour l'analyse, on a utilisé tout d'abord des grandeurs adimensionnelles

$$
\frac{P t h-P_{E}}{\rho \omega^{3} R_{2}^{5}} \quad \text { et } \quad \frac{Q}{\omega R_{2}^{3}},
$$

puis, plus simplement, les valeurs relatives

$$
\frac{P t h-P_{E}}{P_{M}} \text { et } \frac{Q}{Q_{M}},
$$

ce qui conduit à écrire pour la plage de débit considéré.

$$
\frac{P t h-P_{E}}{P_{M}}=A-B \ln \left(\frac{Q}{Q_{M}}\right)
$$

où $A$ et $B$ sont des constantes dépendant de la pompe.

D'autres analyses de courbes faites par ailleurs ont donné en général la même allure logarithmique, même en partant de courbes Hth expérimentales légèrement incurvées vers le haut entre $Q_{M}$ et $Q_{K}$, et en utilisant d'autres modes extrapolation que la droite $H_{E}$ de la figure 10, seules les valeurs de $A$ et $B$ se trouvant modifiées.

Ceci tient, sans doute; à ce que les variations des ordonnées de la courbe $H t h(Q)$ dans sa partie gauche l'emportent de beaucoup sur celles de $H_{E}$ quelles que soient ces dernières. 


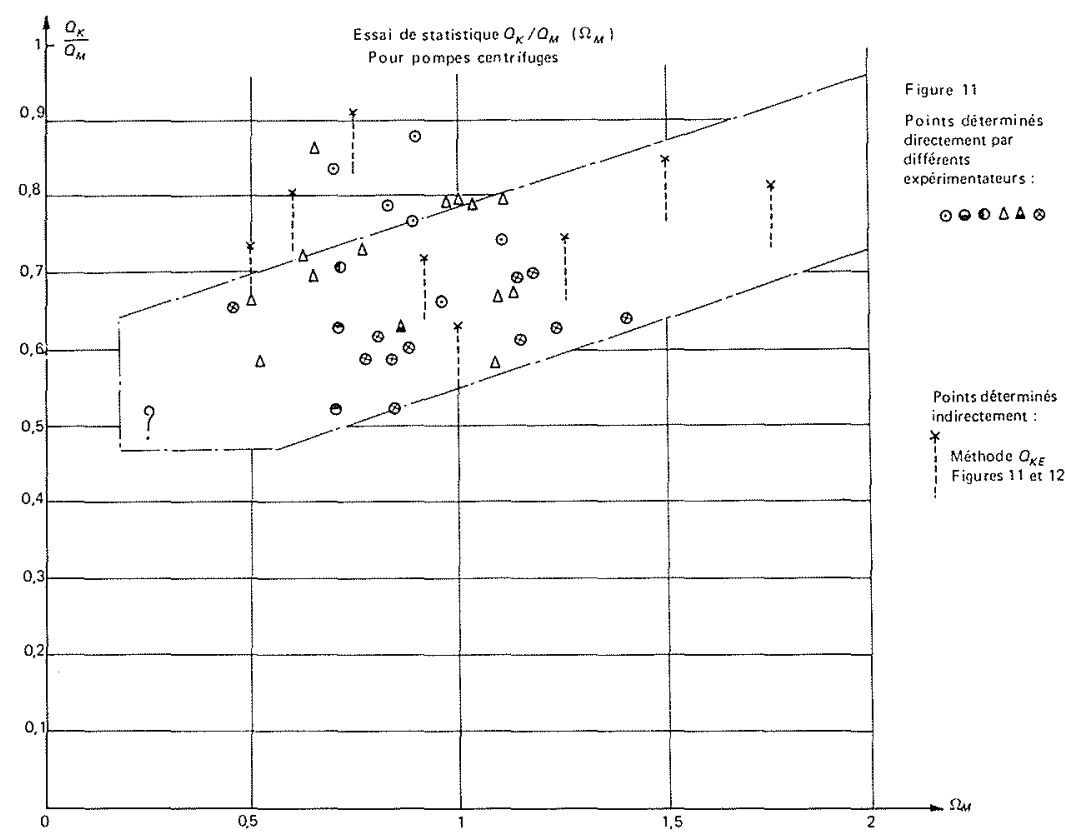

Figure 11 - Essai de statistique $Q_{K} / Q_{M}=f\left(\Omega_{M}\right)$ pour pompes centrifuges

$\odot \diamond \Delta \Delta \otimes$ Points déterminés directement par différents expérimentateurs

$\times$ Points déterminés indirectement - Méthode $Q_{K E}$ figure 11 et 12 .

Cette loi logarithmique reste à expliquer physiquement, quoiqu'il en soit, à partir des points de la figure 11, on peut facilement déterminer le débit $Q_{K_{E}}$ conduisant à Pth $-P_{E}=0$ d'après cette formule en écrivant $Q_{K E} / Q_{M}=e^{A / B}$, les constantes $A$ et $B$ étant déduites par régression linéaire des points de la figure, soit plus simplement en traçant au mieux la droite correspondante en diagramme semilogarithmique.

Bien évidemment, ce point $Q_{K_{E}}$ correspond à un débit un peu plus petit que celui qu'implique le raccordement tangent des figures 9 (2) et 10. Mais comme cette méthode à l'avantage de la simplicité et que, comme nous le verrons, elle donne pour d'autres raisons des valeurs, en général, par excés par rapport à celles déterminées par des techniques directes, nous confondrons ici $Q_{K_{E}}$ et $Q_{K}$.

Variations de $Q_{K} / Q_{M}$ avec $\Omega_{M}$ et $\psi_{M}$ et influence sur les courbes caracteristiques. Tendance à la limitation d'emploi a débit partiel des pompes rapides

Dans l'ensemble, l'expérience industrielle montre que les fonctionnements à débit partiel des pompes centrifuges sont d'autant plus génants que leur rapidité spécifique $\Omega_{M}$ est plus grande.

Cela tient beaucoup à ce que la puissance dissipée à débit partiel prend une importance d'autant plus grande que $\psi_{M}$ diminue et que par la suite $\Omega_{M}$ augmente (voir graphiques $7 a, 7 b$ et 4 ).

La dissipation de puissance par recirculations apparaissant d'autant plus vite que le rapport $Q_{K} / Q_{M}$ est plus grand, l'idée générale est que $Q_{K} / Q_{M}$ tend à augmenter avec $\Omega_{M}$. Nous avons cherché à le constater sur un diagramme statistique $Q_{K} / Q_{M}, \Omega_{M}{ }^{(\delta)}$ (voir Fig. 11).

Sur ce diagramme nous avons reporté divers points expérimentaux observés directement par les auteurs ou d'autres membres du sous-groupe $1 c$, ou même publiés dans la littérature technique.

Nous y avons ajouté un certain nombre de points déterminés par la méthode indirecte décrite ci-dessus. Ces derniers points étant, comme nous l'avons dit, en général par excès, le signe correspondant est prolongé vers le bas.

Comme on peut le voir sur la figure 11 , il y a là encore une grande dispersion de l'ensemble des points et la tendance cherchée n'apparaît pas nettement à première vue.

Mais il faut tenir compte qu'une bonne partie des points situés en haut et à gauche du diagramme correspondent à des pompes dont les diffuseurs ou les volutes ont des angles et des sections plus faibles que ce qui correspond à l'optimum de la roue. Ceci permet pour les pompes lentes, de diminuer $Q_{M}$ et $\Omega_{M}$ sans toucher à la roue. Il en résulte donc aussi une augmentation de $Q_{K} / Q_{M}$ et un déplacement du point correspondant vers le haut et la gauche.

En faisant abstraction de ces pompes à roues et diffuseurs "démariés" nous avons pu tracer une bande approximative pour les points $Q_{K} / Q_{M}, \Omega_{M}$ qui a bien une allure ascendante.

Sur la base des graphiques $6 a$ et $6 b$ précédents ainsi que des graphiques $7 a, 7 b, 7 c$ et 11 nous avons tracé

(8) Il nous a manqué certaines indications pour faire le diagramme en $\psi_{M}$ correspond. 
les figures schématiques moyennes $12 a, 12 b, 12 c$ qui montrent bien l'évolution de la puissance dissipée avec $\psi_{M}$ et $\Omega_{M}$.

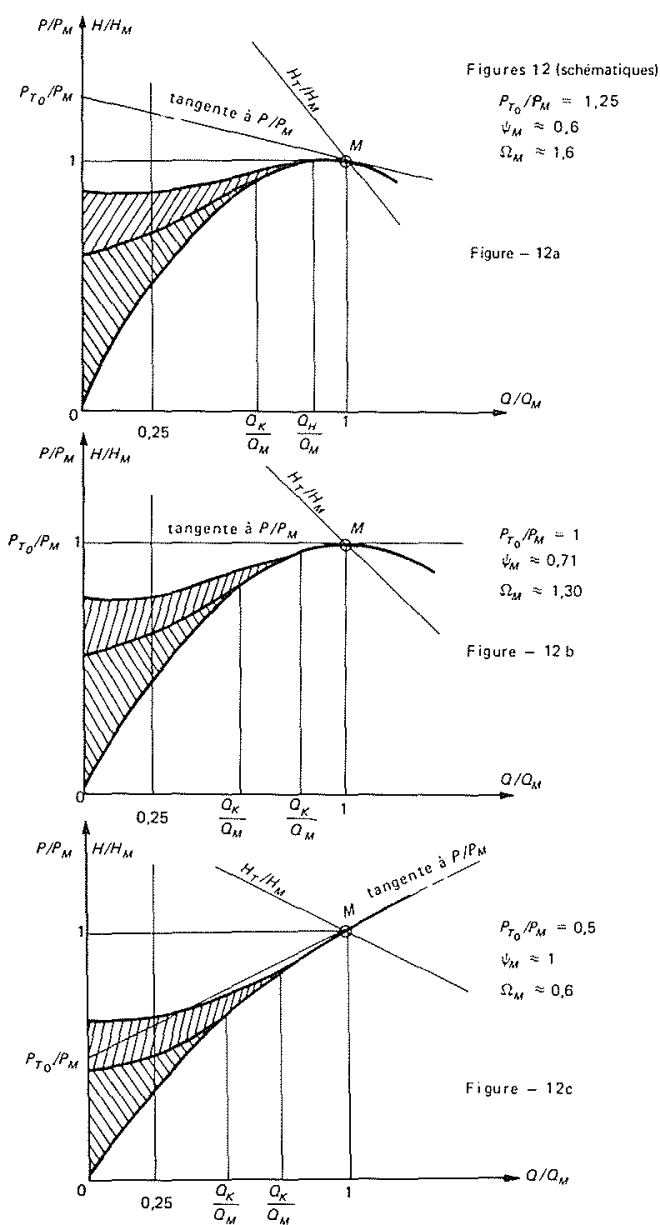

Figure 12 - Evolution de la courbe de puissance avec la rapidité spécifique
a) $P_{T 0} / P_{M}=1,25$
b) $P_{T 0} / P_{M}=1$
$\psi_{M} \approx 0,6$
$\psi_{M} \approx 0,71$
$\Omega_{M} \approx 1,6$
$\Omega_{M} \approx 1,30$
c) $P_{T 0} / P_{M}=0,5$
$\psi_{M} \approx 1$
$\Omega_{M} \approx 0,6$

\section{Remarque}

A ce sujet, il est nécessaire de rappeler que la difficulté d'utilisation à débit partiel des pompes rapides ne vient pas uniquement de l'importance relative de la puissance dissipée. Elle tient aussi à ce que les altérations des courbes caractéristiques, ainsi que les fluctuations de pression et toutes les perturbations qui, y sont liées, y prennent une importance relative plus grande et ceci pour diverses raisons du reste complexes, sortant du cadre de cet exposé.

\section{Conclusions}

A ce qui est dit dans le résumé, on doit ajouter ceci :

1) La façon dont a été faite la statistique a incité tous les constructeurs à présenter non seulement des caractéristiques "satisfaisantes" mais d'autres qui ne l'étaient pas. Ceci a permis en usant de signes appropriés de délimiter pour les paramètres caractéristiques à débit partiel, des zones dont on ne peut sortir qu'avec prudence.

2) Dans les divers graphiques, l'utilisation des valeurs relatives, telle que $P_{0} / P_{M}, Q_{K} / Q_{M}$, etc, a paru plus utile à l'usage que d'autres valeurs adimensionnelles et l'emploi du coefficient $\psi_{M}$ plus fructueux que celui de $\Omega_{M}$.

3) Les pentes relatives des tangentes en $M$ aux courbes $H / H_{M}\left(Q / Q_{M}\right)$ et $P / P_{M}\left(Q / Q_{M}\right)$ nous ont paru être des paramètres importants.

4) Une détermination approximative de $Q_{K} / Q_{M}$ à partir de la courbe $P(Q)$ est possible et utile en l'absence de valeur expérimentale. La méthode proposée pourrait sûrement être perfectionnée.

5) La valeur de $Q_{K} / Q_{M}$ est sans doute importante, mais n'est pas à elle seule un critère de bon fonctionnement d'une pompe centrifuge à débit partiel.

\section{Notations}

\section{Indices}

$M$ à trait au point de rendement global maximal

$K$ à trait au point d'apparition des recirculations

1 à trait à l'entrée de la roue

2 à trait à la sortie de la roue

$Q \quad$ débit volumique de la pompe

$\rho \quad$ masse volumique du liquide pompé

$H$ hauteur totale faite par la pompe

$g \quad$ accélération de la pesanteur

\section{Grandeurs}

(Toutes les grandeurs $G$ hydrauliques ou mécaniques de cet exposé sont en réalité des moyennes temporelles $\bar{G}$ en régime établi. Le signe ${ }^{-}$a été abandonné pour simplifier l'écriture). unité

$\left(\mathrm{m}^{3 / \mathrm{s}}\right)$

$\left(\mathrm{kg} / \mathrm{m}^{3}\right)$

(m)

$\left(\mathrm{m} / \mathrm{s}^{2}\right)$ 
$P \quad$ puissance absorbé sur l'arbre

$\eta \quad=\frac{(P Q)(g H)}{P}=$ rendement de la pompe

$\omega \quad$ vitesse angulaire de la roue

vitesse de rotation de la pompe

$\Omega_{M} \quad$ rapidité spécifique de la pompe

$\Omega_{M}=\frac{\omega \sqrt{Q_{M}}}{\left(g H_{M}\right)^{3 / 4}} \approx 0,01889 \cdot n_{\mathrm{sM}}$

$n_{\mathrm{sM}} \quad$ vitesse spécifique de la pompe $n_{\mathrm{sM}}=\frac{n \sqrt{Q_{M}}}{\left(H_{M}\right)^{3 / 4}}$

$R \quad$ rayons, $R_{2}=\frac{D_{2}}{2}=$ rayon extérieur de l'aubage de la roue

$b_{2} \quad$ largeur de sortie de la roue

$U_{2} \quad=\omega R_{2}=$ vitesse périphérique de l'aubage

$\psi \quad=g H / U_{2}^{2} / 2=$ coefficient de hauteur totale

Hth

(ou $\mathrm{Hi}^{\text {y }}$ hauteur (ou hauteur interne)

$(g H t h)=\frac{P_{r}}{\left(\rho Q_{r}\right)}=$ de masse de liquide la traversant

$J / \mathrm{Kg}$

$P_{r} \quad$ puissance mécanique cédée effectivement à l'aubage de la roue

$$
P_{r}=P-P_{f}
$$

$P_{f} \quad$ puissance absorbée par frottement fluide ou autre sur l'extérieur du rotor

$Q_{r} \quad$ débit volumique traversant la roue

$Q_{f} \quad$ débit de fuite interne retraversant la roue

$$
Q_{r}=Q+Q_{f}
$$

$\eta_{m} \quad=\frac{P_{r}}{P}=\frac{P-P_{f}}{P}=$ rendement mécanique de la pompe d'où $\frac{P_{f}}{P}=\left(1-\eta_{m}\right)$

$\eta_{v} \quad=\frac{Q}{Q_{r}}=\frac{Q}{Q+Q_{f}}=$ rendement volumique de la pompe d'où $\frac{Q_{f}}{Q}=\frac{1}{\eta_{v}}-1 \simeq 1-\eta_{v}$

$\eta_{h} \quad=\frac{H}{H t h}=$ rendement hydraulique de la pompe

$$
\left(\eta=\eta_{h} \times \eta_{v} \times \eta_{m}\right)
$$

$P_{E} \quad$ puissance mécanique à fournir à l'aubage en l'absence de recirculation (suivant modèle d'Euler)

$$
P_{E}=\left(\rho Q_{r}\right)\left(g_{H_{E}}\right)
$$

$H_{E} \quad$ hauteur théorique (ou hauteur interne) en l'absence de recirculation 


\section{Discussion}

\section{Président : R. BRAZZINI}

Sur les interventions de MM. GUITON, SCHIAVELLO et OSTERWALDER, M. REY signale que le débit critique des pompes a été déterminé, soit par les courbes, soit par la méthode des fills colorés au niveau de l'œillard.

M. GUITON précise que plusieurs pompes ne sont pas prises en compte sur la figure 11 dans la bande de dispersion tracée, car elles n'avaient pas de volutes optimales, ce qui peut déplacer sensiblement le point de meilleur rendement pour les pompes à faible vitesse spécifique.

MM. REY et GUITON expliquent qu'il n'a pas été possible comme le préconise M. OSTERWALDER d'analyser en détail les pertes mécaniques (paliers, étanchéités, frottement de disques) de chacune des pompes. Ils précisent de plus que cette méthode est suffisante pour les grosses pompes, leur statistique n'étant valable que pour les pompes d'une puissance supérieure à 50 ou $100 \mathrm{~kW}$.

A la suite d'une question de terminologie de M. BONNIN, le débat s'engage sur la notion đe débit critique.

M. SEDILLE propose une interprétation des phénomènes se produisant dans cette plage de débit. Il faut souligner que le théorème d'EULER s'applique dans tous les cas. Ce qui est discutable par contre, c'est la simplification qui consiste à supposer le mouvement relatif permanent à l'intérieur de la roue alors qu'on sait pertinemment qu'il ne peut pas l'être.

L'écart entre $H$ th et $H_{E}$ reflète l'influence des pulsations dues à cette non permanence et celles-ci existent même en dehors de tous décollement ou inversion de courant. Ces pulsations sont de plus en plus faibles, en valeur relative, au fur et à mesure que le débit moyen augmente, ce qui explique que la courbe $H_{E}$ soit très sensiblement une droite sur une partie de la caractéristique. Il faut toutefois reconnaître qu'un changement de pente brusque de la courbe Hth est dû à un accroissement notable des pulsations, ce qui peut être l'indice d'un décollement ou d'une inversion de courant.

Cependant, cette croissance assez brusque de $H t h$ par rapport à $H_{E}$ existe aussi dans les pompes purement centrifuges à faible nombre de tours spécifiques, à diffuseur et à courbe complètement descendante, pour lesquelles, s'il peut y avoir très probablement des décollements à la sortie des aubes mobiles, il est difficilement concevable qu'elles puissent être le siège d'inversions de courant sur une partie seulement des filets, si l'on admet que ceux-ci sont tous identiques.

Enfin, il faudrait examiner soigneusement dans quelles conditions, pour les pompes à $n_{\mathrm{s}}$ moyen, des inversions de courant à l'entrée peuvent influer sur la courbe Hth qui est plus directement sensible à ce qui se passe à la sortie. Cette influence est certaine pour les machines à très fort $n_{\mathrm{s}}$ telles que les machines axiales; elle n'est pas évidente pour les autres.

M. GUITON fait référence dans la figure 11 au débit critique expérimental d'aspitation, c'est-à-dire au débit pour lequel se produit une inversion locale de la vitesse au niveau de l'œillard.

M. BORCIANI présente deux photographies de l'écoulement dans l'oillard d'une pompe-turbine qui montrent qu'existent deux régimes d'écoulement très différents pratiquement en correspondance avec le débit critique. Les deux régimes donnent lieu à deux courbes séparées qui sont influencées par le coefficient de cavitation : le passage d'une courbe de raccordement à l'autre se produit suivant une coúrbe de raccordement qui est fonction du circuit extérieur. 